\title{
Wireless Power Transfer System For A Human Motion Energy Harvester
}

\author{
Pit Pillatsch ${ }^{\mathrm{a}}$, Eric M. Yeatman ${ }^{\mathrm{b}}$, Andrew S. Holmes ${ }^{\mathrm{b}}$, Paul K. Wright ${ }^{\mathrm{a}}$ \\ ${ }^{a}$ Department of Mechanical Engineering, University of California - Berkeley, CA, USA \\ ${ }^{b}$ Department of Electrical and Electronic Engineering, Imperial College London, UK
}

\begin{abstract}
Human motion energy harvesting as an alternative to battery powering in body worn and implanted devices is challenging during prolonged periods of inactivity. Even a buffer energy storage system will run out of power eventually if there is no external acceleration to the harvester. This paper presents a method to actuate the rotor inside a previously presented rotational piezoelectric energy harvester wirelessly via a magnetic reluctance coupling to an external driving rotor with one or more permanent magnet stacks attached. This makes it possible to recharge a battery or super-capacitor even if a patient is not moving. The use of a permanent magnet coupling has potential advantages compared to traditional inductive or ultrasonic methods, e.g. in terms of tissue damage and transmission depth. Simulation results show the achievable coupling torque for different configurations of magnet geometries and relative positions between the driving magnet stack(s) and the harvester. It is shown that using a single magnet stack yields better results than using two diametrically opposite stacks. Measurements are performed with different magnets, driving frequencies and orientations of the harvester. The results are discussed and successful energy transfer was achieved regardless
\end{abstract}


of the orientation of the device with respect to gravity, which is desirable for real world applications. Lateral misalignment between the harvester and the driving magnet can also be overcome. The largest distance of power transfer reached was $32 \mathrm{~mm}$ with the largest magnets tested, and the optimal power output into a resistive load was over $100 \mu \mathrm{W}$ at a frequency of $25 \mathrm{~Hz}$. The functional volume of the harvester is $1.85 \mathrm{~cm}^{3}-$ similar to the size of a wristwatch.

Keywords: piezoelectric, energy, harvester, rotational, wireless, transfer

\section{Introduction}

The advent of more and more body mounted and implantable devices and sensors raises the question of how to sustainably provide power to them. Ubiquitous computing and body sensor networks, along with the internet of things, are major topics of discussion. Many technologies exist to make these a reality in terms of sensing and monitoring in healthcare as well as industrial settings. However, for truly powerful networks, large numbers of individual devices are required and the common bottleneck is the energy source. There are many applications where primary and secondary batteries are successful and appropriate. The constant need for recharging batteries in portable electronics is certainly a nuisance, but it is not a crucial drawback that hampers widespread adoption. Ultra-low-power electronics and communications can help mitigate the issue. It seems to be the case however that while many sensors, such as accelerometers in smart phones, are seeing increased energy efficiency, the addition of new sensors and communications drives the power consumption back up. Ultimately, efforts should be made on both ends of 
the scale, supply and demand, in order to find viable solutions.

Energy harvesting, the local generation of electricity from surrounding sources, is becoming an option for human body applications [1, 2, 3, 4, 5]. Especially in the case of implanted devices, a perpetual power source would be highly beneficial as it could extend the lifetime of implants and eliminate the need for intrusive surgery. In addition, a large number of failures in medical devices are caused by the batteries [6, 7]. Suitable sources for human energy harvesting are temperature differences [8], photovoltaic systems $[9,10]$ and glucose fuel cells [11]. Body motion is an abundant source of energy that can work for externally worn and implanted devices alike $[12,13,14]$. A good overview of the available solutions for powering medical devices can be found in [15]; [16] provides an introduction with a focus on piezoelectric devices and [17] is more specific to electromagnetic power generation.

One question that remains regarding all body mounted vibrational energy harvesting is what happens if the wearer does not move for extended periods of time, e.g. when bed-bound due to illness. It is realistic to assume that harvesters will have some form of backup or buffer energy storage, such as a super-capacitor or rechargeable battery, to overcome relatively short bouts of inactivity, for example at night during sleep. However, in cases of prolonged rest, there is a severe risk of the implant failing. Previously, the authors introduced a piezoelectric energy harvester for human body motion with a rotational proof mass [18]. Rotational harvesters are less dependent on their orientation with respect to gravity than linear devices where the proof mass can get stuck at an end stop. The device also employs the principle of frequency up-conversion through contact-less magnetic plucking of a piezo- 
electric beam. This technique, where the transducer always operates at its ideal frequency, has become popular for dealing with low frequency, random excitation. An electromagnetic version of such a mechanism was presented in [19]. Impact driven devices as in [20, 21, 22, 23] bear the risk of damaging the brittle piezoelectric ceramic material in the long term. A more promising approach appears to be plucking via plectra $[24,25,26]$. The contact-less magnetic coupling chosen by the authors has also been employed by others [27].

The previously presented prototype operates similarly to a self-winding wristwatch and thus uses a proof mass close in dimensions to the rotor in a Seiko Kinetic watch [28]. It has been shown in [29] that an external driving magnet can latch on to the internal rotor of the device through a magnetic reluctance coupling. This adds the capability of externally actuating the harvesting mechanism and to generate electricity through use of a dedicated source in the absence of external excitation from motion. It is assumed that during normal operation, i.e. when a person is not bed bound, the power generated from inertial energy harvesting is sufficient to keep the system running and to trickle charge a buffer storage capacitor or battery. The addition of wireless charging capability provides a non-intrusive option of power delivery that significantly reduces the burden on a patient. This paper describes the wireless power transfer system and presents simulation and experimental results. 


\section{Magnetic reluctance coupling to the energy harvester}

Figure 1 shows the components of the harvester as it was introduced in [18]. Operating as a harvester, the semi-circular rotor can accept linear and rotational inertial excitation. As the rotor moves, a small permanent magnet swipes past a second permanent magnet that is attached to the tip of a piezoelectric bimorph beam. The repelling magnet force causes the beam tip to deflect up to the point where the beam force exceeds the magnetic forces and the beam is released to vibrate at its natural frequency. Given the high natural frequency of the beam, an up-conversion from the low input excitation frequency is thus achieved, rendering the transduction more efficient.

The reluctance coupling to the internal rotor is illustrated in figure 2 . The rotor is a half disc made of magnetic mild steel. The discontinuity at the edge of the half disc causes a change in reluctance when a permanent magnet is moved past it from the outside. The resulting magnetic force $F_{\text {mag }}$ creates a torque that can be used to actuate the rotor during periods of rest, when no inertial forces on the rotor are present. The direction of polarisation of this driving permanent magnet is different from the one of the tip magnet on the beam. As a result, no significant adverse effects of the driving magnet onto the tip magnet were experienced during the measurements.

Wireless RF energy harvesting and power transfer, as described in [30, 31, 32], differ by the fact that harvesting utilises ambient RF energy, while transfer employs a dedicated source. The latter is the case for the device presented in this paper. Human body applications requiring wireless power transfer often use inductive or ultrasonic coupling [33, 34, 15, 35]. Potential 
drawbacks of inductive coupling are safety concerns due to the high power high frequency fields, and increasing tissue attenuation at higher frequencies which limits the penetration depth [36]. This is the reason why electrodynamic receivers have been investigated. The principle relies on coupling the magnetic field from a transmitter coil to a permanent magnet in a receiver at a distance. The resulting motion of the permanent magnet then induces a current in a surrounding coil, rather than the coil picking up the magnetic field from the transmitter directly $[37,38,39,40]$. Another variant of this principle uses a rotating magnet receiver [41].

The system in this paper is different in that it uses a magnetic reluctance coupling, where a permanent magnet couples on to a semi-circular steel rotor. Permanent magnetic torque couplings or gears in general have the advantage that no lubrication is required and that they can transfer torque where a separation of media is needed $[42,43,44]$. They are used in applications such as waste water pumps. Through-wall transmission using alternating magnetic fields has been demonstrated [45]. One device for powering a pacemaker that uses permanent magnetic coupling in a similar way to this work is introduced in $[46,47]$. The differences are that an electromagnetic transduction mechanism is used instead of a piezoelectric one and that the device in itself can not function as a standalone energy harvester. In addition, due to the specific arrangement of the coupling and the transduction mechanism it was shown that the magnetic field from the coupling negatively affected the transduction. 


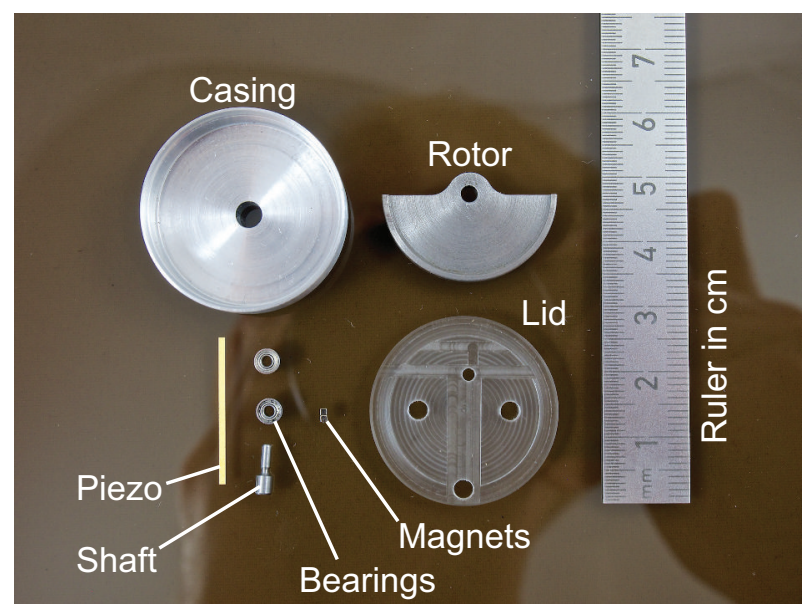

Figure 1: Piezoelectric frequency up-converting rotational energy harvester components

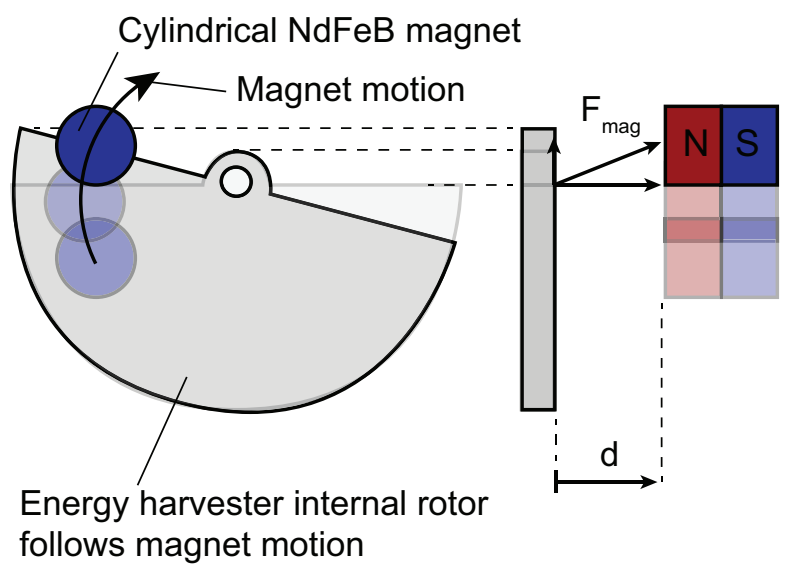

Figure 2: Magnetic reluctance coupling of an external driving magnet to the internal harvester rotor 


\section{Steady state simulation of the achievable coupling torque}

In order to better understand the coupling of an external magnet to the rotor, a series of Comsol 5.0 FEM simulations was performed. The simplified model contains the imported geometry of the mild steel rotor and three different geometries of permanent magnets as listed in table 1, corresponding to the magnet stacks used for the experiments outlined below. The material for the rotor is defined as soft iron and the magnetic saturation is defined by the corresponding $\mathrm{BH}$-curves using the $\mathrm{AC} / \mathrm{DC}$ module. The permanent magnets are characterised via their residual flux density $B_{r}\left(B_{r}=1.31 \mathrm{~T}\right.$ for rectangle magnets and $B_{r}=1.465 \mathrm{~T}$ for both cylindrical magnets) and relative permeability $\mu_{r}=1.05$.

The cylindrical coordinate system as used for the simulations is shown in figure 3. The advantage of this definition is that the main parameters of interest can easily be investigated via a parametric sweep of the relative position between the magnet and the rotor. The sweep is run across the angle from the edge of the rotor $\varphi$, the distance $r$ from its axis of rotation and the separation distance $d$ in $z$-direction. The generated coupling torque $T_{z}$ around the $z$-axis is ultimately the result of interest as it determines whether the coupling is strong enough for the rotor to follow the motion of the external driving magnet.

One result of the magnetic flux density from the simulation is shown in figure 4 for the case of small cylinder magnets with $\varphi=7.5^{\circ}, d=1 \mathrm{~mm}$, $r=12 \mathrm{~mm}$. The flux density on the surface of the NdFeB magnet reaches about $1.4 \mathrm{~T}$, which is consistent with the value assumed for $B_{r}$. It can be seen that the rotor material saturates in the corner of the half disk, reaching 
about 2.2 $\mathrm{T}$ which is the upper limit prescribed by the BH-curve for the soft iron material definition.

The first sweep of simulations was performed for a stack of small cylinder magnets and with a broad overview in mind. Each parameter was varied over 7 values: $\varphi= \pm 15^{\circ}, r=4-21 \mathrm{~mm}, d=1-16 \mathrm{~mm}$. This results in 343 individual simulations being solved to determine the coupling torque $T_{z}$. Figure 5 shows the result in relation to $d$ and $\varphi$ at a fixed value of $r=12 \mathrm{~mm}$. First, the torque decreases rapidly with the distance $d$ between the magnet and rotor, as is to be expected. Due to this result, the sweep over the parameter $d$ was omitted in subsequent simulations to reduce the processing effort. The resolution over the other two parameters $\varphi$ and $r$ was increased instead. Second, the results indicate that at $r=12 \mathrm{~mm}$, a positive angle $\varphi=5-10^{\circ}$ yields optimal torque.

The reason for choosing $r=12 \mathrm{~mm}$ in figure 5 becomes clear when it is evaluated in combination with $T_{z}$ as a function of $\varphi$ and $r$ as shown in figure 6. At this radial distance the torque is maximised. The radius of the rotor is $13.5 \mathrm{~mm}$ and so a radial distance that is too large will reduce the coupling force whereas a radial distance that is too small will reduce the leverage that creates the torque.

After acquiring these initial findings, a more detailed parametric sweep was performed, keeping a constant $d=1 \mathrm{~mm}$ and varying $\varphi= \pm 20^{\circ}, r=$ $10-14 \mathrm{~mm}$. The optimum of $r=12 \mathrm{~mm}$ is fairly consistent across all values of $\varphi$, with $\varphi=7.5^{\circ}$ yielding the maximum torque. In practical terms, $r$ is a parameter that can be optimised, whereas the system will settle on the ideal value of $\varphi$ on its own. It is nevertheless interesting to investigate a 
range of values for $\varphi$ as the results show that there are very few cases where $T_{z}(\varphi)$ assumes negative values, regardless of the position of the magnet on either side of the edge of the rotor, i.e. positive or negative values of $\varphi$. This behaviour explains why the addition of a diametrically opposite stack of magnets is detrimental to the system performance as will be further discussed in the experimental section below. If one magnet at a certain angle $\varphi$ exerts a positive torque $T_{z}(\varphi)$, then a diametrically opposite one exerts a negative torque $-T_{z}(-\varphi)$. These contributions are not equal and opposite because the function $T_{z}(\varphi)$ is not even. Consequently, there is a net positive torque but it is reduced by the second magnet stack.

Figures 7 and 8 show the simulation results for the large cylinder and rectangular magnets respectively. The range of parameters is $\varphi=-20^{\circ}-$ $+20^{\circ}$ and $r=5-16 \mathrm{~mm}$. The results are very similar to the previous case with smaller cylinder magnets in that the ideal value for $r$ is still around $11-12 \mathrm{~mm}$ but the optimal $\varphi$ is now $+15^{\circ}$ for large cylinders whereas it is still $+7.5^{\circ}$ for the rectangular magnets. Again, the torque rarely reaches negative values and the maximally achievable values for all three magnet geometries are in line with the expectation that the largest magnets provide the largest torque. 
Table 1: Parameters of the permanent magnets used in the experiments and simulations

\begin{tabular}{l|l|l} 
Designation & Dimensions & Magnetization \\
\hline \hline Small cylinders & $1 / 4 \times 3 / 8 " \quad$ (diameter $\times$ thick- & N52 \\
ness $)$ & \\
Large cylinders & $\begin{array}{l}3 / 8 \times 3 / 8 " \quad(\text { diameter } \times \text { thick- } \\
\text { ness }) \\
\text { Rectangles }\end{array}$ & $\begin{array}{l}\text { N52 } \\
\text { width } \times 1 / 8 \times 1 / 4 " \text { length })\end{array}$
\end{tabular}

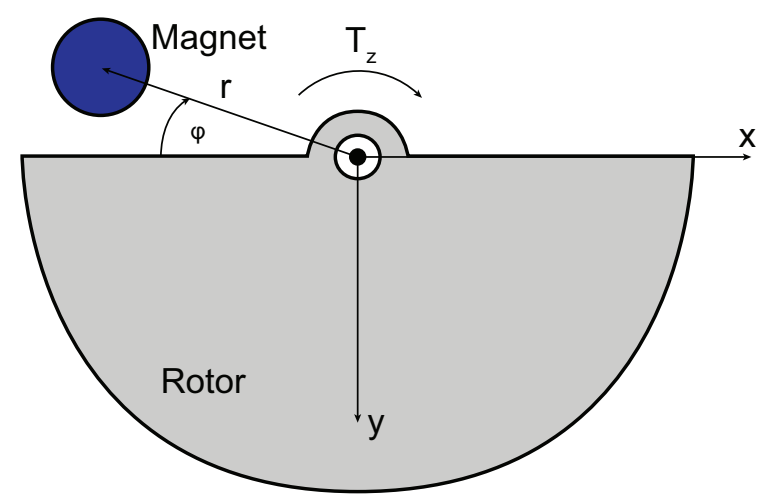

Figure 3: Coordinate system for the Comsol simulations, the torque transferred around the z-axis, $T_{z}$, is the main result of interest 


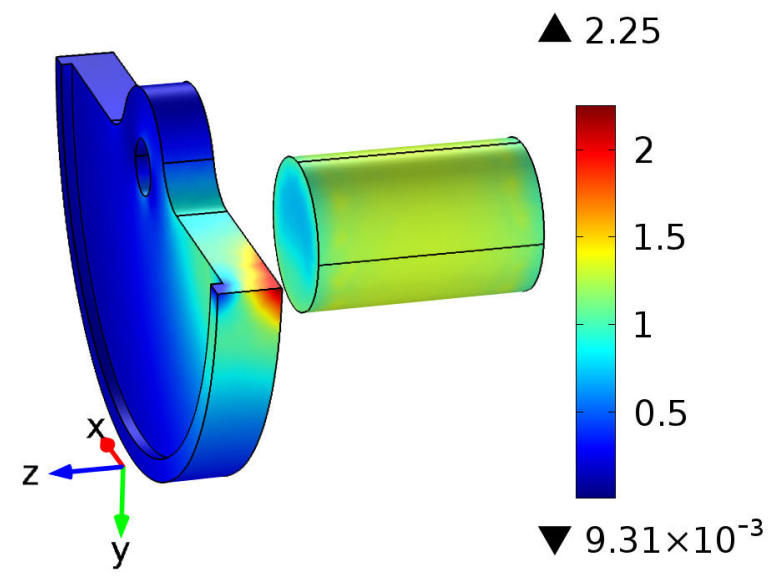

Figure 4: Simulated magnetic flux density in Tesla for parameters $\varphi=7.5^{\circ}, d=1 \mathrm{~mm}$, $r=12 \mathrm{~mm}$

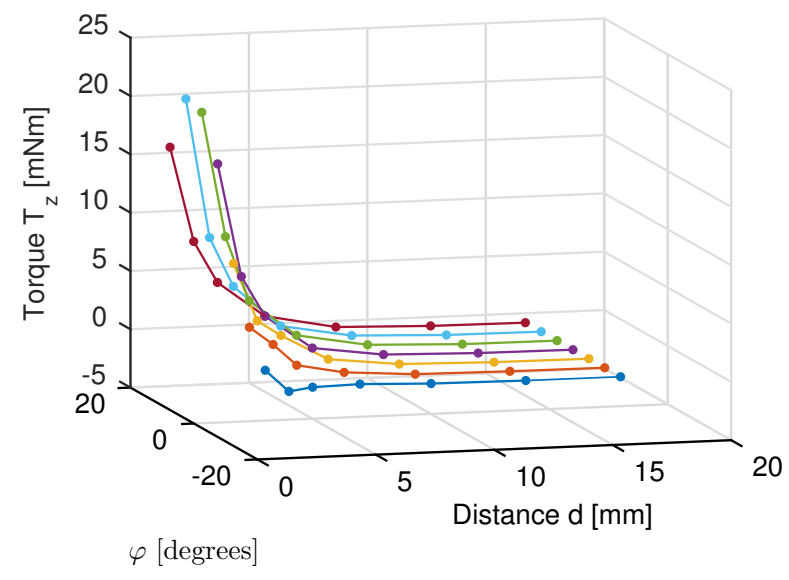

Figure 5: Simulated coupling torque $T_{z}$ in relation to separation distance $d$ and angle $\varphi$ for small cylinder magnets 


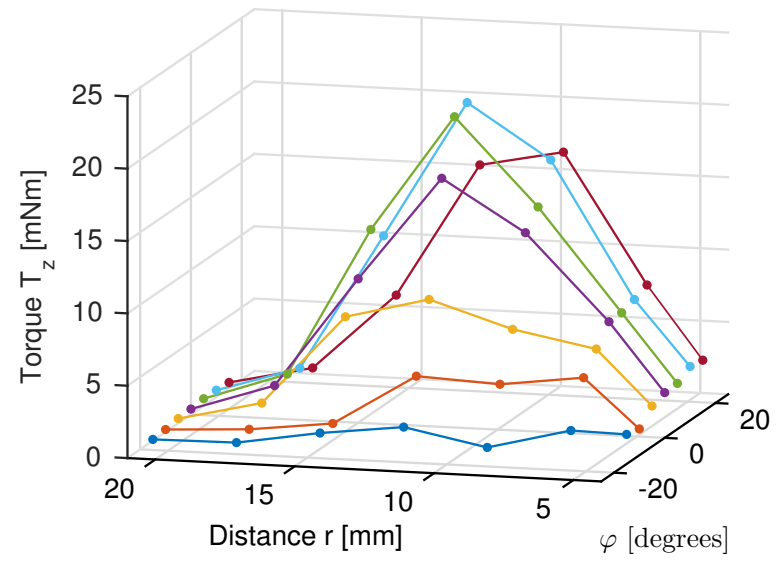

Figure 6: Simulated coupling torque $T_{z}$ in relation to radial distance $r$ and angle $\varphi$ for small cylinder magnets

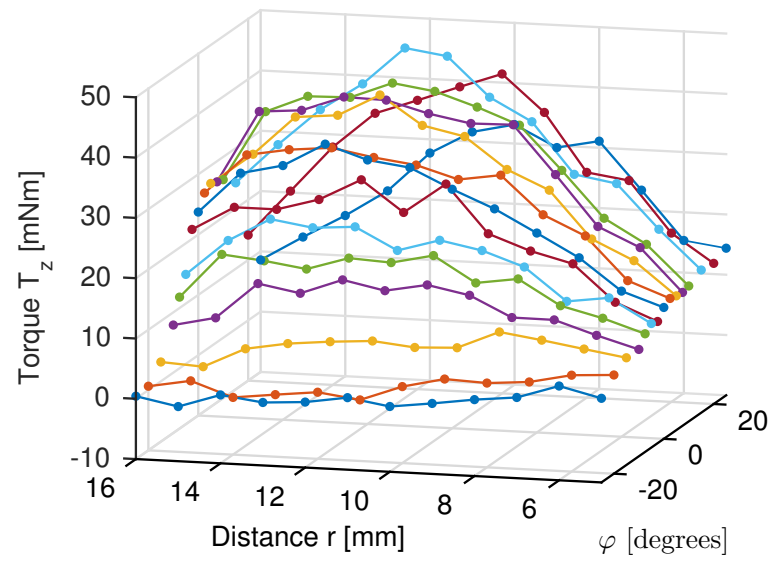

Figure 7: Simulated coupling torque $T_{z}$ in relation to radial distance $r$ and angle $\varphi$ for large cylinder magnets 


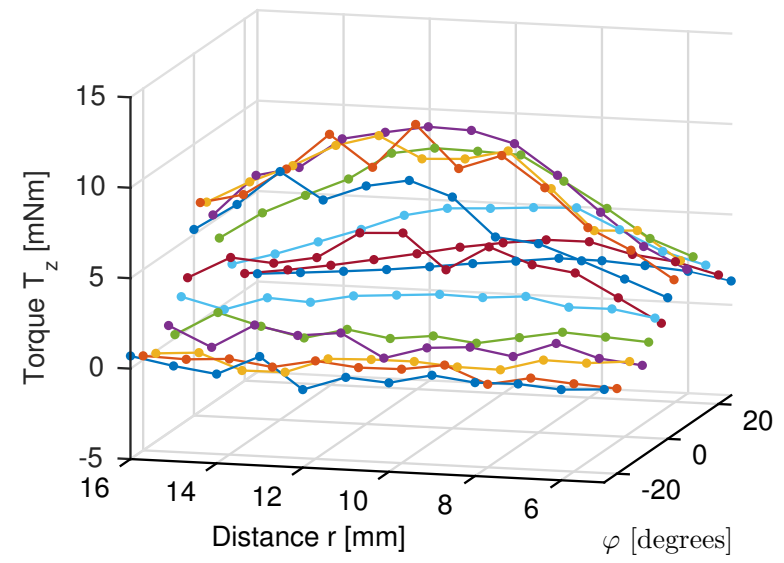

Figure 8: Simulated coupling torque $T_{z}$ in relation to radial distance $r$ and angle $\varphi$ for rectangular magnets 


\section{Experimental set-up and procedure}

Figure 9 shows the experimental set-up. The energy harvester is clamped in the left mount, which is movable in the direction of the black ruler at the bottom. This is used to vary the distance $d$ between the external driving magnet and the harvester. Two wires extend outwards from the piezoelectric beam inside the harvester casing and are connected to an oscilloscope either with or without an impedance-matched load of $150 \mathrm{k} \Omega$ attached. The power output is determined as squared voltage drop divided by the resistance. Three consecutive measurements of rms voltage are taken over a period of $10 \mathrm{~s}$ each and then averaged to minimise the measurement error. It is worthwhile to note that the energy harvester does not include any power conditioning circuitry or back-up battery in this case. Measuring the power output across a resistive load makes it possible to acquire the raw power output without any losses from regulators and rectifiers. The error bars are only shown in the graphs where appropriate as they are in general insignificantly small.

The right mount carries a stepper motor of type 3303 from Phidgets Inc. that is driven by a matching 1067 stepper motor control circuit. This combination allows for a higher rotational speed of up to $4800 \mathrm{rpm}$ (or $80 \mathrm{~Hz}$ ) than is normally possible with a stepper motor. This is achieved through micro-stepping and ramping of the acceleration. Expressed as a frequency $f$ in Hertz, the rotational velocity matches the plucking frequency of the piezoelectric beam. The benefit of using a stepper motor over a regular DC motor is that the frequency can be easily and precisely controlled and consistent speed is better maintained. During all experiments with a constant

driving frequency, e.g. for investigating the effects of separation distance $d$, 
the stepper motor was left running while changing parameters. This has an effect on the results as will be described below. In contrast to the left mount, the right mount allows for displacement along the silver ruler on the right hand side to investigate the effects of lateral misalignment $L$.

Three different orientations are considered as depicted in figure 10. Experiments are carried out with the piezoelectric beam inside the harvester pointing either up or down in a vertical arrangement, or in a horizontal arrangement. In vertical orientation, gravity affects the rotor inside the harvester. When the piezoelectric beam is pointing down, it is plucked at the stable bottom equilibrium point under gravitational force. In contrast, when the beam is pointing up, it is plucked at the unstable equilibrium. In a horizontal configuration, gravitational forces act in the direction of the rotor axis and do not have any effect. This range of experiments gives insight into the system behaviour under different orientations as would occur in human body applications. In addition, some experiments are carried out with two diametrically opposite stacks of magnets, as photographed in figure 9, or with only one stack of magnets in a one sided, asymmetric configuration. The magnets, according to the dimensions in table 1, are made up of stacks of three or four individual magnets, where the bottom one is pressed into the 3d-printed carrying disk. The initial experiments were performed with small cylinder stacks that were located at a radial distance $r=10.5 \mathrm{~mm}$. Based on the simulation results described in the previous section, the radial distance for the experiments with large cylinders and rectangles was increased to $r=12 \mathrm{~mm}$, as this yields optimum coupling torque $T_{z}$.

A complete description of the energy harvester is given in [18], where 
the operation of the device in different orientations while it is worn is also outlined. In comparison, the various orientations described in the context of the wireless power transfer mechanism are aiming at relative positioning between the harvester and the driving magnet while the wearer is at rest. The most relevant parameters are the harvester casing diameter of $30 \mathrm{~mm}$ and functional volume of $1.85 \mathrm{~cm}^{3}$. The piezoelectric beam is a series connected bimorph with a free length of $19.5 \mathrm{~mm}, 1 \mathrm{~mm}$ wide and $0.37 \mathrm{~mm}$ thick.

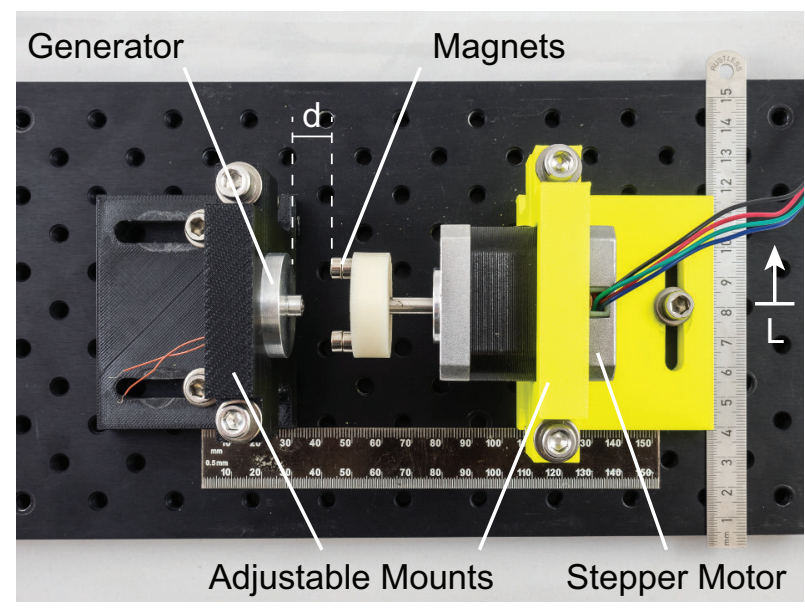

Figure 9: Experimental set-up, adjustable mounts are used to set different values of separation distance $d$ and lateral misalignment $L$

\section{Measurement results}

The first series of experiments investigates the power output when varying the distance $d$ between the harvester and the driving magnet. The driving frequency was chosen to be $10 \mathrm{~Hz}$ and the stepper motor was left running while changing the distance $d$. Figure 11 shows the results for small cylinder magnets. Several characteristic behaviours can be be observed. The power 

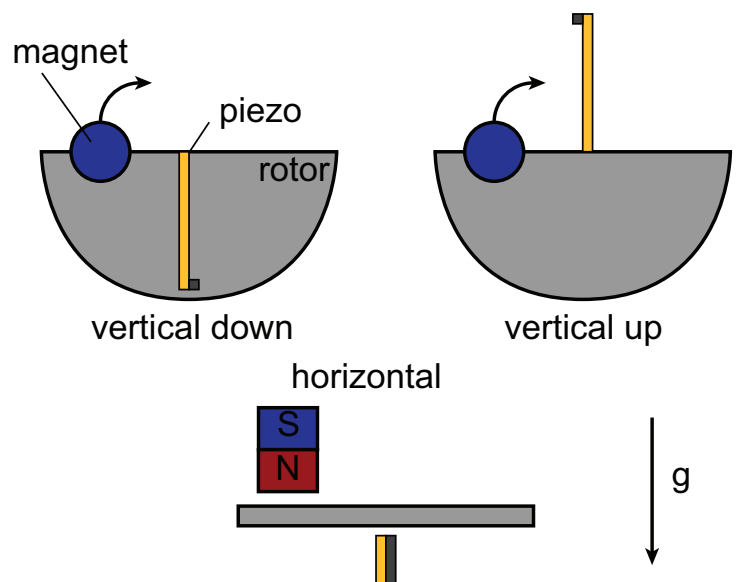

Figure 10: Harvester and piezoelectric beam orientations considered during the experiments to investigate the influence of gravity

output is slightly scattered at close proximity between the harvester and external magnets but stays level afterwards in all investigated orientations. It is expected that the power output stays level. As long as the torque coupling is strong enough for the rotor to follow the driving magnets, the rotational frequency, and consequently the number of beam actuations, will remain constant. At a certain point, the coupling is too weak, the rotor stops, and the power output abruptly drops to zero.

In the configuration with a single magnet stack, this drop-off occurs earliest when the beam is facing up at about $d=6 \mathrm{~mm}$, then at $d=18 \mathrm{~mm}$ with the beam facing down and only at $d=20 \mathrm{~mm}$ in horizontal arrangement. The power output follows a different pattern, being lowest with the beam facing up and highest with the beam facing down, reaching close to $50 \mu \mathrm{W}$. Both these phenomena can be explained by the influence of gravity. In the horizontal arrangement, the coupling torque $T_{z}$ only has to overcome the beam actuation force whereas in vertical arrangements the gravitational 
forces on the rotor need to be overcome as well. This explains the higher separation distance achievable in a horizontal arrangement. In addition, when the beam is facing down, gravity helps accelerate the rotor towards the beam tip, resulting in a higher passing velocity between the rotor and the beam and thus a stronger actuation and the highest power output. Without gravity, the horizontal arrangement ends up in the middle in terms of power output and when the beam is facing up, gravity slows the rotor down until it overcomes the unstable equilibrium at the top causing the slowest passing velocity and lowest power output. The general behaviour is very similar in the case with two diametrically opposite stacks of magnets. The arrangement with two magnet stacks generally achieves smaller separation distance $d$ and the explanation was in part discussed based on the simulation results. The second stack of magnets will cancel out the torque $T_{z}$ to a certain extent because of the symmetry with respect to the rotor half disc edge and the fact that $T_{z}$ generally stays positive regardless of the angle $\varphi$. The exception is the orientation with the beam facing up, where two magnet stacks actually achieve higher separation distance. This behaviour is not entirely understood but it might be influenced by the fact that in a vertical orientation, the pendulum resonance frequency, calculated as $5.6 \mathrm{~Hz}$ plays a role. Nevertheless, in general there are no benefits in using two stacks of magnets, which is why this configuration was no longer considered for further experiments.

The corresponding voltage output measured across the load resistor on the piezoelectric beam is shown in figure 12. The number of actuations of the piezoelectric beam matches the $10 \mathrm{~Hz}$ driving frequency of the external magnets exactly. Each actuation causes an initial deflection followed by a 
ring down of the beam at its natural frequency.

Figures 13 and 14 are from the same experiment for large cylinder and rectangular magnets respectively. The results regarding maximal power output and achievable separation distance between the three orientations are consistent with the previous findings. As is to be expected, large cylinder magnets can achieve a very large separation distance of up to $32 \mathrm{~mm}$. Rectangular magnets achieve very similar separation distances to small cylindrical magnets with very level power outputs across the range of tested distances and a high maximal separation even in the case with the beam pointing up.

In order to conclude the investigation of separation distance, a series of experiments was performed when stopping the driving motor each time before changing $d$ for large cylinder magnets. The graph in figure 15 shows that this dramatically lowers the achievable separation distance and the coupling between rotor and magnets. It is assumed that in the previous experiments, the inertia of the rotor, once it is set in motion at a small $d$, extends the operable range of the system. In practical terms, this means that an initial shaking of the harvester (e.g. moving the arm for an implanted device) might be needed in order for the rotor to couple on to the driving magnet and follow its movement if the separation distances are larger.

Overall, the results from the first series of experiments demonstrate that power transfer can be achieved successfully regardless of the orientation of the device, despite the fact that a horizontal arrangement that is not affected by gravity is in general preferable. In addition, rectangular magnets yield more consistent performance in all scenarios.

The power output is mostly determined by the frequency of actuation 
of the internal piezoelectric beam. The power output in relation to driving frequency can be seen in figure 16 for a fixed distance $d=1 \mathrm{~mm}$. The graph shows that it follows the same curve for all tested orientations, in this case with the small cylinder magnets. One would expect it to increase linearly. However, there is an optimum around $25 \mathrm{~Hz}$. This phenomenon is explained by an additional experiment carried out up to a frequency of $50 \mathrm{~Hz}$ in the vertical configuration with the beam pointing down. The corresponding figure 17 illustrates the recorded voltage at that frequency and offers an explanation for the drop in power output. At such high frequencies, the free oscillation of the beam has not died off yet before the next actuation happens. This causes a complicated interaction between the beam tip that is still vibrating and the approaching rotor and ultimately lowers the initial deflection of the next beam actuation. At the optimal driving frequency, a power output of over $100 \mu \mathrm{W}$ is achieved.

In a real world application, where the location of the harvester might not be known exactly, it is of interest to know how a lateral misalignment $L$ between the axis of the harvester and the axis of the stepper motor affects the power transfer. The experiments depicted in figure 18 were performed at a separation $d=1 \mathrm{~mm}$ and frequency $f=10 \mathrm{~Hz}$ at which a misalignment of $L=4 \mathrm{~mm}$ was reached before the small cylinder magnets would collide with the protruding bearings of the current version of the harvester casing. This is the only experiment where the errorbars have been included in the graph, as the results are more scattered than in previous experiments. Nevertheless, the general behaviour is consistent with the previous results, where the vertical arrangement with beam pointing down offers the highest 
power output, followed by the horizontal arrangement and then the one with the beam pointing up. The power output is hardly affected by this slight misalignment, which is a welcome result.

The corresponding voltage output in figure 19 at a value of $L=4 \mathrm{~mm}$ shows that under misalignment, the rotor occasionally misses a turn before latching onto the driving magnet again. This explains the more significant error bars in figure 18 .

Lastly, an interesting phenomenon was observed in the open circuit voltage at $d=1 \mathrm{~mm}$ and at low driving frequency $f=5 \mathrm{~Hz}$ with a single stack of small cylinder magnets in figure 20. Regular "bumps" show up as an overlay of the decaying voltage of the piezoelectric beam at a time of $0.1 \mathrm{~s}$ and every $0.1 \mathrm{~s}$ thereafter. If this was due to the larger external driving magnet having an effect on the internal beam tip magnet, the effect would be visible at the driving frequency of $5 \mathrm{~Hz}$ rather than twice that frequency as is the case. It is assumed that the internal semi-circular rotor inside the harvester has become magnetized due to the interaction with the external magnets. Consequently, the passing edges of the rotor create a small actuation force on the beam tip magnet. 

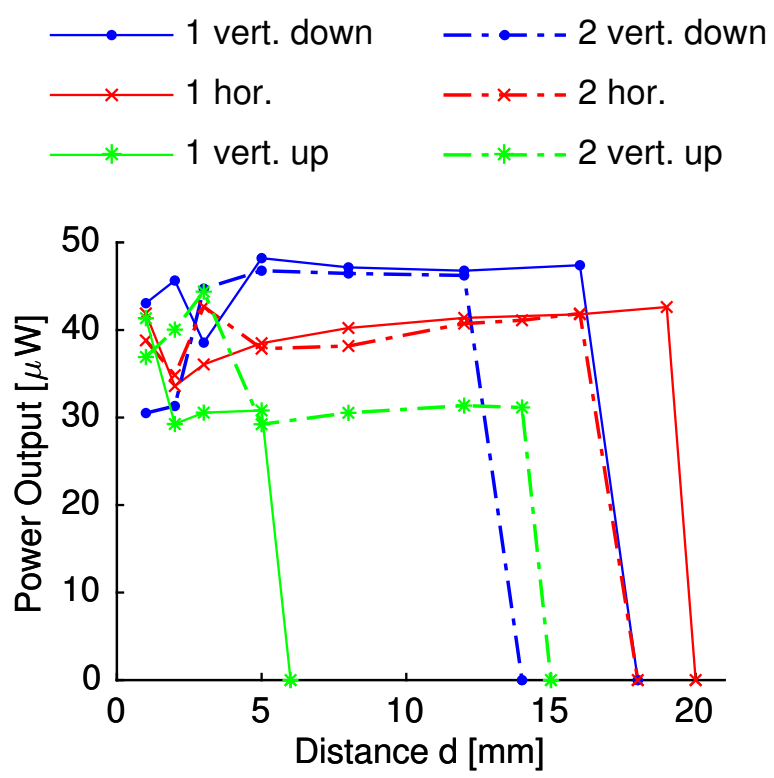

Figure 11: Experimental power output in relation to separation distance $d$ with small cylinder magnets

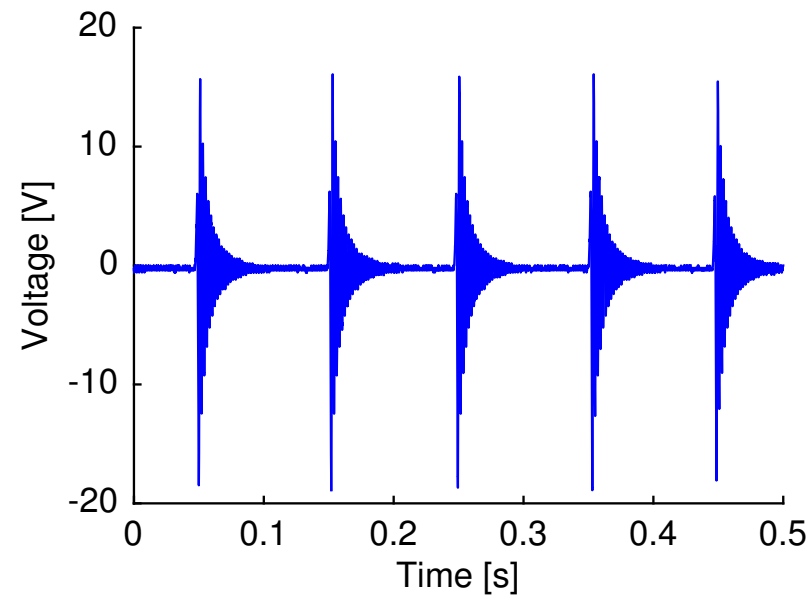

Figure 12: Experimentally recorded voltage at a frequency of $f=10 \mathrm{~Hz}$ 

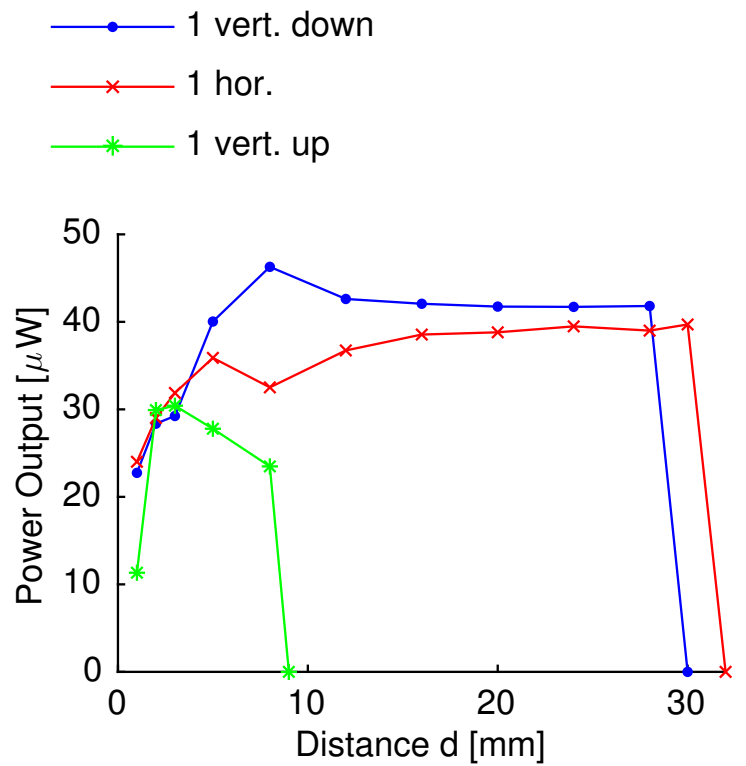

Figure 13: Experimental power output in relation to separation distance $d$ with large cylinder magnets 

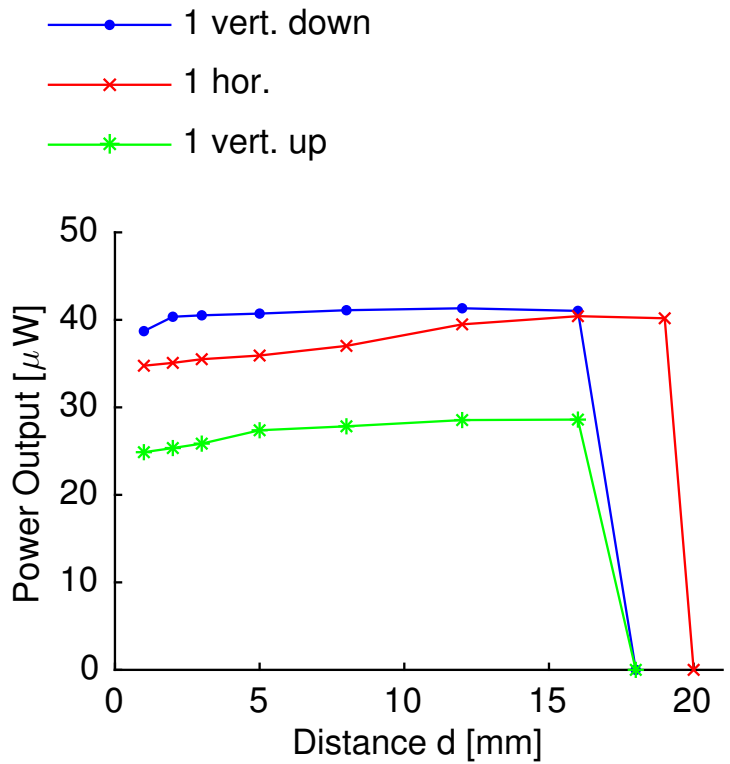

Figure 14: Experimental power output in relation to separation distance $d$ with rectangular magnets 

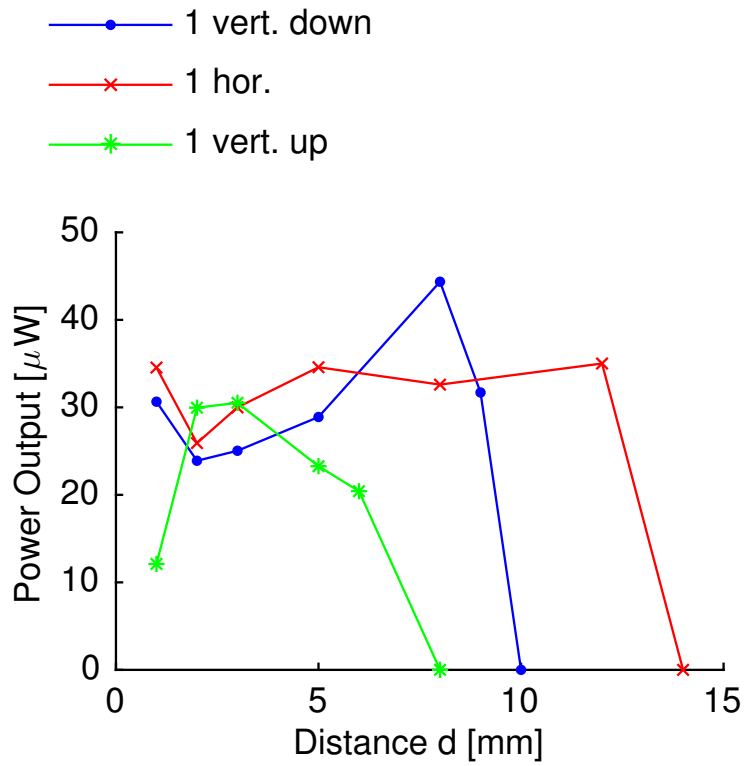

Figure 15: Experimental power output with large cylinder magnets when the driving magnet is stopped between each adjustment of the separation distance $d$ 

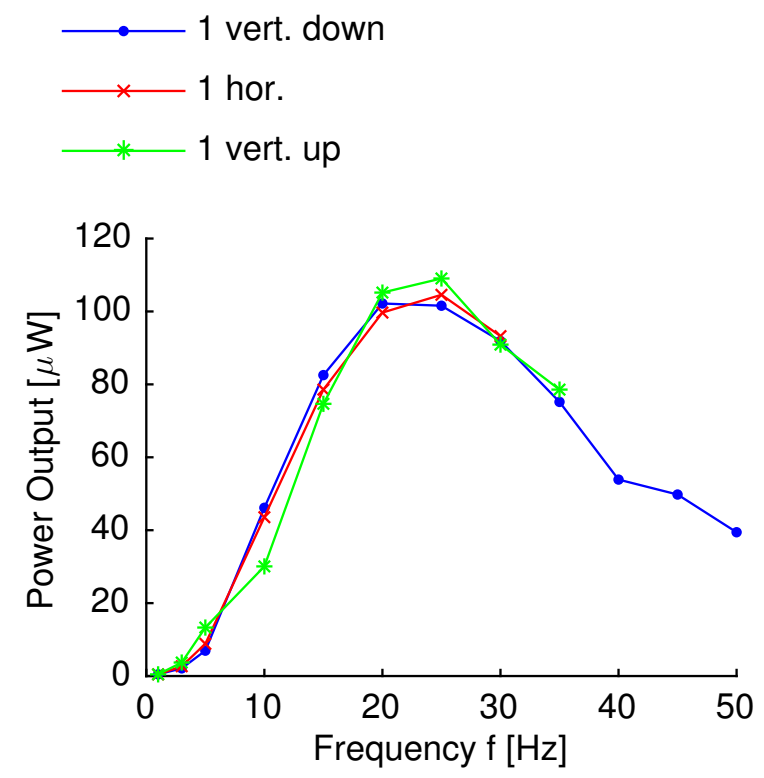

Figure 16: Experimental power output in relation to driving frequency $f$ with small cylinder magnets

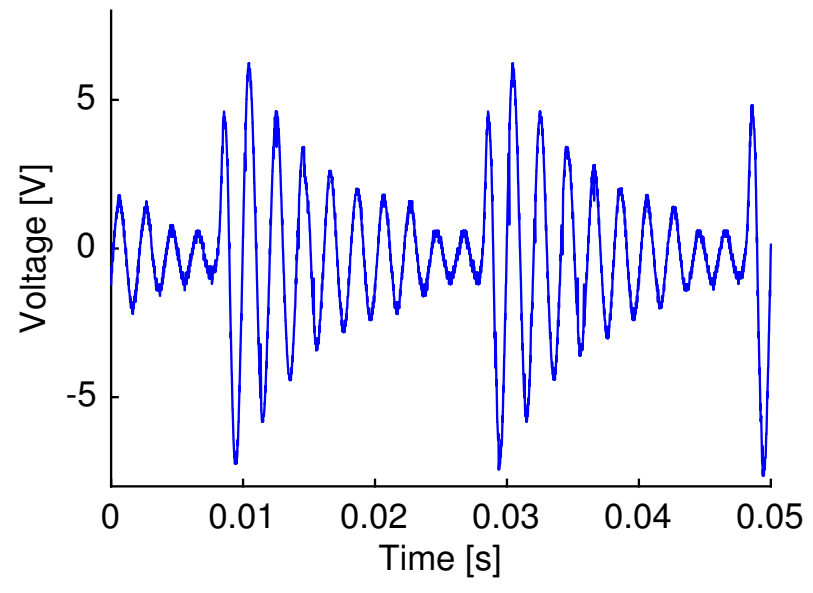

Figure 17: Experimentally recorded voltage at a frequency of $f=50 \mathrm{~Hz}$ 


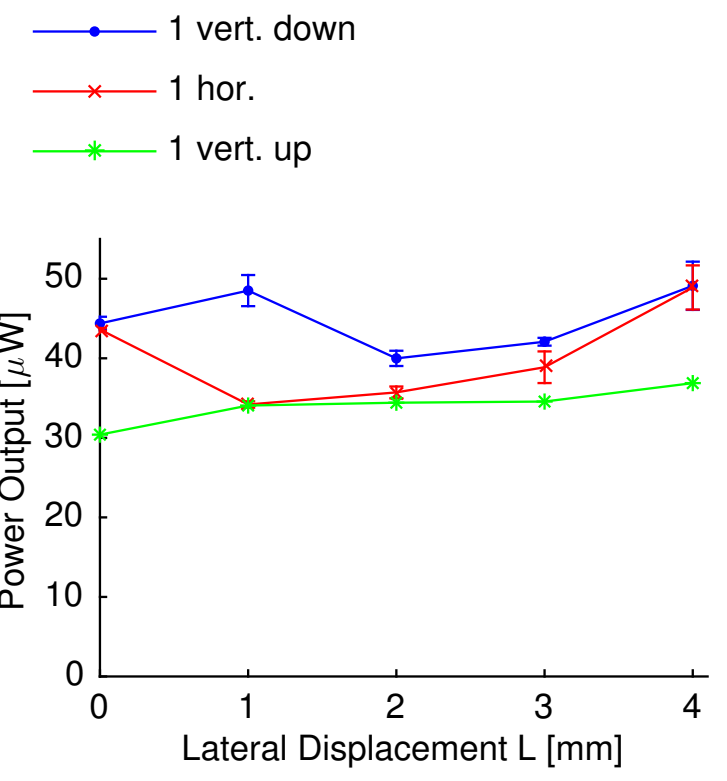

Figure 18: Experimental power output in relation to lateral misalignment $L$ with small cylinder magnets

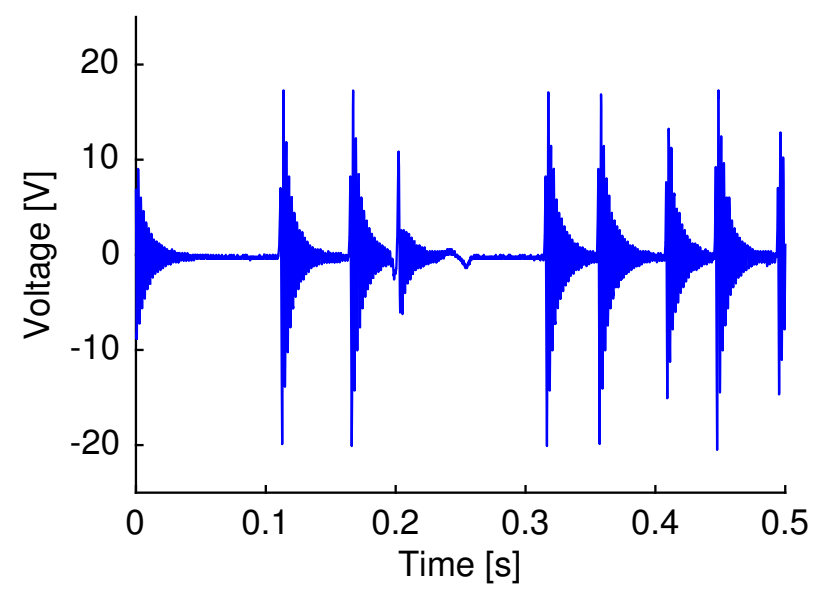

Figure 19: Experimentally recorded voltage across and impedance-matched resistive load at a frequency of $f=10 \mathrm{~Hz}$ and lateral misalignment $L=4 \mathrm{~mm}$ 


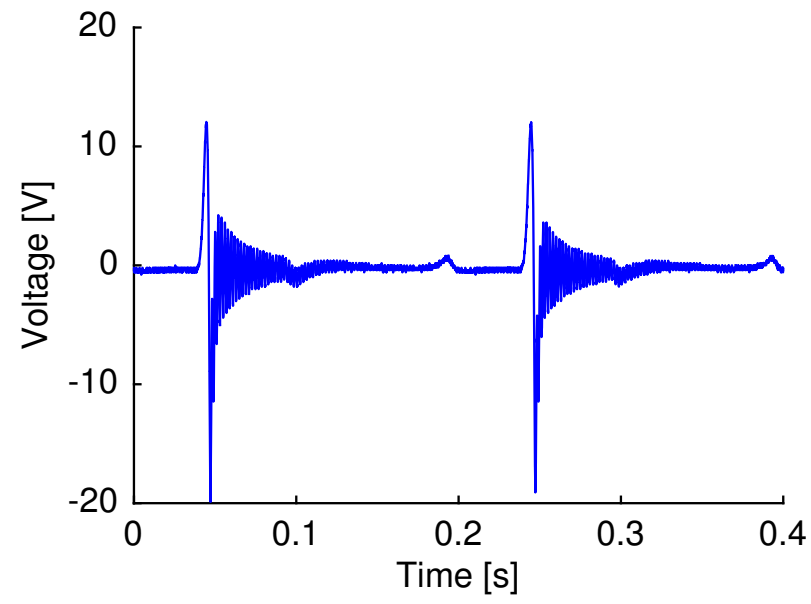

Figure 20: Experimentally recorded open circuit voltage at $f=5 \mathrm{~Hz}$ 


\section{Conclusions}

This paper presents a wireless energy transfer mechanism adding the capability of charging a buffer energy storage to a human motion energy harvesting device during periods of rest, when the lack of external excitation would otherwise cause the system to fail. The energy harvester operates similarly to the generator in a self-charging wristwatch. An eccentric rotor made of mild steel actuates a piezoelectric beam and the system relies on frequency up-conversion through magnetic plucking for improved conversion effectiveness. Without any alterations to the harvester itself, wireless energy transfer can be achieved via a magnetic reluctance coupling between an external driving magnet and the internal rotor that follows the motion of this magnet.

Comsol simulation results of this reluctance coupling are presented and the optimal values for the radial distance between the driving magnet and the axis of the rotor are discussed. Regardless of the relative angle $\varphi$, the coupling torque $T_{z}$ generally assumes positive values, which explains why the addition of a diametrically opposite second stack of driving magnets is actually detrimental to the performance. The torque generated from the second stack of magnets opposes the one from the first stack and reduces the overall torque. Three different geometries of driving magnets are investigated and it is found that the ideal radial distance is very similar in each case. As is to be expected, larger magnets create a stronger coupling torque which allows for larger separation distances $d$ between the harvester and the driving magnet.

The results from the simulations are verified through experiments. The 
measurement set-up comprises a stepper motor for the driving magnets and adjustable mounts used for varying the separation distance $d$ and the lateral misalignment $L$. Experiments are performed with single and dual magnet stacks, different magnet geometries and under different orientations with and without the effects of gravity. The results demonstrate successful wireless power transfer regardless of the orientation of the harvester which is beneficial for real world applications. It is also concluded that there is an optimal driving frequency of about $25 \mathrm{~Hz}$ at which over $100 \mu \mathrm{W}$ of power were dissipated across an impedance-matched resistive load. Beyond this frequency, the free beam oscillations have not fully rung down before the next actuation occurs and the resulting interactions between beam tip and rotor magnet reduce the power output. The largest distance of transfer was achieved with large cylinder magnets and reached $32 \mathrm{~mm}$ in a horizontal orientation where gravity does not have an effect on the harvester rotor. It is likely that even larger distances could be achieved and one of the potential benefits of this method is that this could cause less damage to the tissue compared to the more traditional inductive coupling.

Finally, this method of wireless energy transfer can be a valuable addition to a pre-existing motion energy harvester, eliminating the major concern of failure at rest. The use of this technique is not limited to human applications and it could be applied in areas that require separation between harsh environments or general through-wall wireless power transfer. 


\section{Acknowledgements}

The present project is supported by the National Research Fund, Luxembourg.

\section{References}

[1] Paul D Mitcheson, Eric M Yeatman, G Kondala Rao, Andrew S Holmes, and Tim C Green. Energy Harvesting from Human and Machine Motion for Wireless Electronic Devices. In Proc. of the IEEE, volume 96, pages 1457-1486, 2008.

[2] Paul D Mitcheson. Energy harvesting for human wearable and implantable bio-sensors. Annual International Conference of the IEEE Engineering in Medicine and Biology Society, 1:3432-3436, January 2010.

[3] T Starner. Human-powered wearable computing. IBM Systems Journal, 35:1-12, 1996.

[4] E Romero, R O Warrington, and M R Neuman. Energy scavenging sources for biomedical sensors. Physiological Measurement, 30(9):R3562, September 2009.

[5] Thomas von Büren, Paul D Mitcheson, Tim C Green, Eric M Yeatman, Andrew S Holmes, and Gerhard Tröster. Optimization of Inertial Micropower Generators for Human Walking Motion. IEEE Sensors Journal, 6(1):28-38, 2006.

[6] William H Maisel, Megan Moynahan, Bram D Zuckerman, Thomas P Gross, Oscar H Tovar, Donna-bea Tillman, and Daniel B Schultz. Pace- 
maker and ICD Generator Malfunctions - Analysis of Food and Drug Administration Annual Reports. Journal of the American Medical Association, 295(16):1901-1906, 2006.

[7] A Lodder, M. V. Kamath, A. R. Upton, and D Armstrong. Evaluation of the Efficacy and Performance of Medical Implants: A Review. Journal of Long-Term Effects of Medical Implants, 20(3):173-185, 2010.

[8] Vladimir Leonov, Chris Van Hoof, and Ruud J.M. Vullers. Thermoelectric and Hybrid Generators in Wearable Devices and Clothes. In 2009 Sixth International Workshop on Wearable and Implantable Body Sensor Networks, pages 195-200. IEEE, June 2009.

[9] JA Paradiso and T Starner. Energy scavenging for mobile and wireless electronics. Pervasive Computing, IEEE, 2005.

[10] S. Roundy, E.S. Leland, J. Baker, E. Carleton, E. Reilly, E. Lai, B. Otis, J.M. Rabaey, V. Sundararajan, and P.K. Wright. Improving Power Output for Vibration-Based Energy Scavengers. IEEE Pervasive Computing, 4(1):28-36, January 2005.

[11] Pratyush Rai, Thang Ho, Jining Xie, Jamie a. Hestekin, and Vijay K. Varadan. Glucose Driven Nanobiopower Cells for Biomedical Applications. Journal of Nanotechnology in Engineering and Medicine, 1(2):021009, 2010.

[12] Jaeseok Yun, Shwetak N Patel, Matthew S Reynolds, and Gregory D Abowd. Design and Performance of an Optimal Inertial Power Harvester 
for Human-powered Devices. IEEE Transactions on Mobile Computing, 8(7):1-16, 2010.

[13] Yuan Rao, Kelly M McEachern, and David P Arnold. A compact human-powered energy harvesting system. Journal of Physics: Conference Series, 476:012011, 2013.

[14] Yuan Rao, Shuo Cheng, and David P Arnold. An energy harvesting system for passively generating power from human activities. Journal of Micromechanics and Microengineering, 23(11):114012, 2013.

[15] Xiaojuan Wei and Jing Liu. Power sources and electrical recharging strategies for implantable medical devices. Frontiers of Energy and Power Engineering in China, 2(1):1-13, March 2008.

[16] K A Cook-Chennault, N Thambi, and A M Sastry. Powering MEMS portable devices - a review of non-regenerative and regenerative power supply systems with special emphasis on piezoelectric energy harvesting systems. Smart Materials and Structures, 17(4):043001 (33pp), August 2008.

[17] David P. Arnold. Review of microscale magnetic power generation. IEEE Transactions on Magnetics, 43(11):3940-3951, 2007.

[18] Pit Pillatsch, Eric M. Yeatman, and Andrew S. Holmes. A piezoelectric frequency up-converting energy harvester with rotating proof mass for human body applications. Sensors and Actuators A: Physical, 206:178185, October 2013. 
[19] Tzeno Galchev, Hanseup Kim, and Khalil Najafi. Micro Power Generator for Harvesting Low-Frequency and Nonperiodic Vibrations. Journal of Microelectromechanical Systems, 20(4):852-866, 2011.

[20] Lei $\mathrm{Gu}$ and Carol Livermore. Impact-driven, frequency up-converting coupled vibration energy harvesting device for low frequency operation. Smart Materials and Structures, 20(4):045004 (10pp), April 2011.

[21] E Jacquelin, S Adhikari, and M I Friswell. A piezoelectric device for impact energy harvesting. Smart Materials and Structures, 20(10):105008 (12pp), October 2011.

[22] M Renaud, P Fiorini, R van Schaijk, and $\mathrm{C}$ van Hoof. An Impact Based Piezoelectric Harvester Adapted to Low Frequency. In TRANSDUCERS 2009 - 2009 International Solid-State Sensors, Actuators and Microsystems Conference, pages 2094-2097, 2009.

[23] Huicong Liu, Chengkuo Lee, Takeshi Kobayashi, Cho Jui Tay, and Chenggen Quan. Piezoelectric MEMS-based wideband energy harvesting systems using a frequency-up-conversion cantilever stopper. Sensors and Actuators A: Physical, pages 1-7, February 2012.

[24] Michele Pozzi, Min S H Aung, Meiling Zhu, Richard K Jones, and John Y Goulermas. The pizzicato knee-joint energy harvester: characterization with biomechanical data and the effect of backpack load. Smart Materials and Structures, 21(7):075023 (8pp), July 2012.

[25] J. Rastegar. Piezoelectric-based power sources for harvesting energy from platforms with low-frequency vibration. Proc. SPIE 6171, Smart 
Structures and Materials 2006: Industrial and Commercial Applications of Smart Structures Technologies, 6171, 2006.

[26] A M Wickenheiser and E Garcia. Broadband vibration-based energy harvesting improvement through frequency up-conversion by magnetic excitation. Smart Materials and Structures, 19(6):065020 (11pp), June 2010.

[27] Q C Tang, Y L Yang, and Xinxin Li. Bi-stable frequency up-conversion piezoelectric energy harvester driven by non-contact magnetic repulsion. Smart Materials and Structures, 20(12):125011 (6pp), December 2011.

[28] Seiko Corp. PARTS CATAlOGUE / TEChNiCAL GUIDE Cal. $5 \mathrm{M} 62 \mathrm{~A}, 5 \mathrm{M} 63 \mathrm{~A}$.

[29] P Pillatsch. Wireless Energy Transfer Through Magnetic Reluctance Coupling. Journal of Physics: Conference Series, 557:1-5, 2014.

[30] Hubregt J. Visser and Ruud J. M. Vullers. RF Energy Harvesting and Transport for Wireless Sensor Network Applications: Principles and Requirements. Proceedings of the IEEE, 101(6):1410-1423, June 2013.

[31] Manuel Piñuela and Paul D Mitcheson. Ambient RF Energy Harvesting in Urban and Semi-Urban Environments. IEEE Transactions On Microwave Theory And Techniques, 61(7):2715-2726, 2013.

[32] André Kurs, Aristeidis Karalis, Robert Moffatt, J D Joannopoulos, Peter Fisher, and Marin Soljacic. Wireless power transfer via strongly coupled magnetic resonances. Science (New York, N.Y.), 317(5834):83-86, 2007. 
[33] Shaul Ozeri and Doron Shmilovitz. Ultrasonic transcutaneous energy transfer for powering implanted devices. Ultrasonics, 50(6):556-566, 2010.

[34] Alexey Denisov and Eric Yeatman. Ultrasonic vs . Inductive Power Delivery for Miniature Biomedical Implants. In Body Sensor Networks, pages $2-7,2010$.

[35] Je Dok Kim, Chuanbowen Sun, and In Soo Suh. A proposal on wireless power transfer for medical implantable applications based on reviews. IEEE Wireless Power Transfer Conference 2014, IEEE WPTC 2014, pages 166-169, 2014.

[36] Jose Oscar Mur-Miranda, Shuo Cheng, David P Amoldt, and Franklin W Olin. Improving the Efficiency of Electrodynamic Wireless Power Transmission. In 7th Europ. Conf. on Antennas and Propagation, pages 2848-2852, 2013.

[37] V. R. Challa, S. Cheng, and D. P. Arnold. The role of coupling strength in the performance of electrodynamic vibrational energy harvesters. Smart Materials and Structures, 22(2):025005, 2013.

[38] Vinod R Challa, V Divakar, Jose Oscar Mur-Miranda, and David P Arnold. Wireless power transmission to an electrodynamic energy harvester using low-frequency magnetic fields. Tech. Dig. 11th PowerMEMS 2011, pages 335-338, 2011.

[39] Vinod R Challa, Jose Oscar Mur-Miranda, and David P Arnold. Wireless power transmission to an electromechanical receiver us- 
ing low-frequency magnetic fields. Smart Materials and Structures, 21(11):115017, 2012.

[40] Shuo Cheng and David P Arnold. Wireless Power Transmission Via Magnetic Coupling To an Electrodynamic Receiver. In Tech. Dig. 10th PowerMEMS 2010, 2010.

[41] A Garraud, J D Jimenez, N Garraud, and D P Arnold. Electrodynamic Wireless Power Transmission to Rotating Magnet Receivers. Journal of Physics: Conference Series, 557:012136, 2014.

[42] E.P. Furlani. A two-dimensional analysis for the coupling of magnetic gears. IEEE Transactions on Magnetics, 33(3):2317-2321, 1997.

[43] Weilai Li. High Efficiency Wireless Power Transmission At Low Freqeuncy Using Permanent Magnet Coupling. PhD thesis, University of British Columbia, 2007.

[44] K. Atallah and D. Howe. A novel high-performance magnetic gear. IEEE Transactions on Magnetics, 37(4 I):2844-2846, 2001.

[45] C. L. Zhang, J. S. Yang, and W. Q. Chen. Through-wall Power Transmission Using an Alternating Magnetic Field and a Multiferroic Energy Harvester. Journal of Intelligent Material Systems and Structures, 22(6):561-565, 2011.

[46] Shin-nosuke Suzuki, Shunsuke Kimura, Tamotsu Katane, Hideo Saotome, Osami Saito, and Kazuhito Kobayashi. Power and Interactive Information Transmission to Implanted Medical Device Using Ultrasonic. 
Japanese Journal of Applied Physics, 41(Part 1, No. 5B):3600-3603, 2002.

[47] Shin-Nosuke Suzuki, Manabu Ishihara, and Yukio Kobayashi. The Improvement of the Noninvasive Power-Supply System. IEEE Transactions on Magnetics, 47(10):2811-2814, 2011. 


\section{Highlights}

- We present amechanism for wireless power transfer to a preexisting human motion energy harvesting device

- Wireless charging can be used to bridge the gap in power generation during prolonged rest periods of the wearer

- Different arrangements of permanent magnets that couple onto the internal proof mass of the harvester are investigated

- Based on findings from simulations the best candidates for the magnet geometry and arrangement are experimentally evaluated

- Power output measurements in a laboratory environment are presented and agree well with the simulation 


\section{Author biographies}

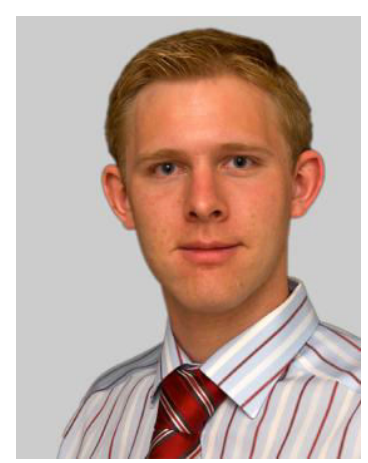

Pit Pillatsch received his BSc. and MSc. degrees in mechanical engineering from the Swiss Federal Institute of Technology, Zurich (ETHZ) in 2008 and 2010 respectively (after completing his MSc. thesis work at the University of Kyoto, Japan). He has received the PhD degree in electrical engineering from Imperial College London in 2014. He is currently the AFR Luxembourg postdoctoral fellow at the Berkeley Energy and Climate Institute at the University of California - Berkeley, where his research is currently focused on efficient energy harvesting, in particular from low frequency and random motion, wireless energy transfer and degradation of piezoelectric materials for energy harvesting.

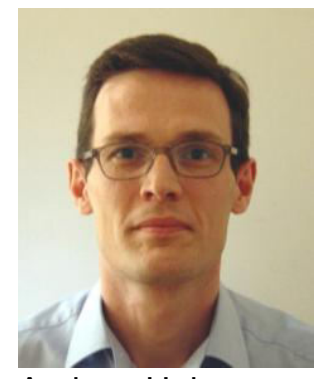

Andrew Holmes received the BA degree in natural sciences from Cambridge University in 1987, and the PhD degree in electrical engineering from Imperial College London in 1992. $\mathrm{He}$ is currently Professor of Micro-Electro-Mechanical Systems (MEMS) in the Department of Electrical and Electronic Engineering, Imperial College London. He has worked on a range of topics in optical signal processing, integrated optics and MEMS, and has published around 150 journal and conference papers in these areas. His current research interests include applications of laser processing in MEMS manufacturing, novel assembly technologies for MEMS and electronics, micro-power generation by energy harvesting, power conditioning electronics for energy harvesters and ultra-low power radio for wireless sensors. He is a cofounder and director of Microsaic Systems plc, an Imperial College spin-out company started in 2001 to exploit Imperial College MEMS research. 


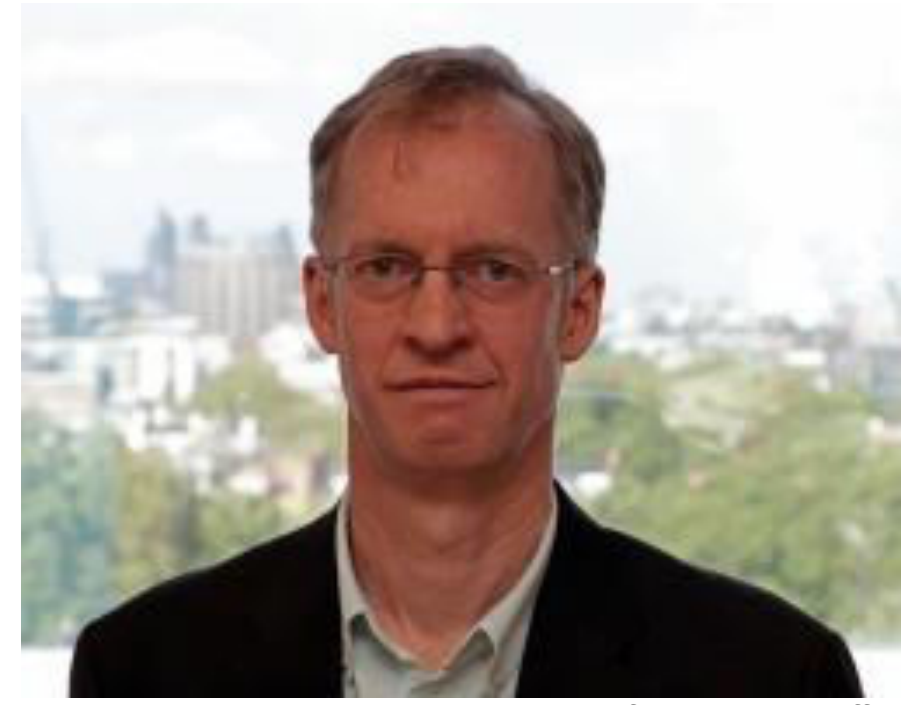

Eric M. Yeatman has been a member of academic staff in Imperial College London since 1989, and Professor of Micro-Engineering since 2005. He has published more than 160 papers and patents, primarily on optical devices and materials, and micro-electro-mechanical systems (MEMS). Prof. Yeatman is also co-founder and director of Microsaic Systems plc, which develops and markets miniature mass spectrometers based on MEMS technology. His current research interests are in energy sources for wireless devices (particularly energy harvesting), radio frequency and photonic MEMS devices, and sensor networks.

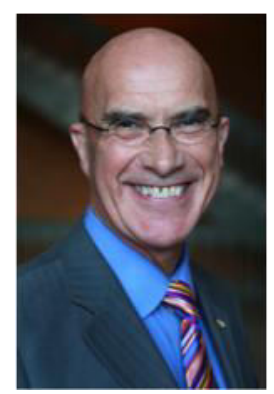

Paul Wright is the A. Martin Berlin Professor of Mechanical Engineering and Director of the Berkeley Energy and Climate Institute. Hls research takes place in the Advanced Manufacturing for Energy (AME) laboratory. Funds from industry, foundations, the federal government, and the California Energy Commission (CEC), support an integrated research program on the resilience \& analytics of energy systems. Individual PhD projects cover a broad spectrum: Communicating MEMS-sensors for advanced electrical-grids and gas distribution systems; Energy harvesting; 3D printing of storage systems; Demand Response, and Condition Based Monitoring (CbM) of energy systems. These projects are the catalysts for start-ups such as Imprint Energy and Wireless Industrial Technologies. 